\title{
Diagnóstico e primeira manifestação de displasia arritmogênica de ventrículo direito em paciente sexagenário
}

\author{
Clinical manifestations and diagnosis of arrhythmogenic \\ right ventricular dysplasia in sexagenary patient
}

\author{
Alexandre de Matos Soeiro ${ }^{1}$, Rodrigo S. Barbosa ${ }^{2}$, Priscila G. Goldstein ${ }^{1}$, Maria Carolina \\ Feres de Almeida ${ }^{3}$, Carlos V. Serrano Jr. ${ }^{4}$, Múcio Tavares de Oliveira Jr. ${ }^{5}$
}

\begin{abstract}
Soeiro AM, Barbosa RS, Goldstein PG, Almeida MCF, Serrano Jr CV, Oliveira Jr MT. Diagnóstico e primeira manifestação de displasia arritmogênica de ventrículo direito em paciente sexagenário / Clinical manifestations and diagnosis of arrhythmogenic right ventricular dysplasia in sexagenary patient. Rev Med (São Paulo). 2014 jan.-mar.;93(1):22-25.
\end{abstract}

RESUMO: A displasia arritmogênica de ventrículo direito (DAVD) é uma das principais causas de morte súbita em indivíduos jovens. Suas manifestações e consequente diagnóstico costumam ser precoces ainda na adolescência ou no adulto jovem. Nesse contexto, o diagnóstico em doente sexagenário sem história familiar sugestiva ou sintomas prévios e com possibilidade de tratamento torna esse caso único na literatura.

DESCRITORES: Displasia arritmogênica ventricular direita; Idoso.

\begin{abstract}
Arrhythmogenic right ventricular dysplasia is one of the major causes of death in younger patients and its manifestations and diagnosis used to be early. In this context, diagnosis in a sexagenary patient without famylial history or previous symptoms and with possibility of correct treatment make this case unique.
\end{abstract}

KEYWORDS: Arrhythmogenic right ventricular dysplasia; Aged.

1. Médico(a) Cardiologista Assistente da Unidade Clínica de Emergência - Instituto do Coração (InCor) do Hospital das Clínicas da Faculdade de Medicina da Universidade de São Paulo (HCFMUSP). E-mail: alexandre.soeiro@cardiol.br.

2. Médico Residente em Cardiologia do InCor-HCFMUSP. Unidade Clínica de Emergência - Instituto do Coração (InCor) do Hospital das Clínicas da Faculdade de Medicina da Universidade de São Paulo.

3. Médica Cardiologista Plantonista da Unidade Clínica de Emergência - InCor - HCFMUSP. Instituto do Coração (InCor) do Hospital das Clínicas da Faculdade de Medicina da Universidade de São Paulo (HCFMUSP).

4. Médico Cardiologista Diretor da Unidade Clínica de Aterosclerose e Professor Livre-Docente - InCor - HCFMUSP. Unidade Clínica de Emergência - Instituto do Coração (InCor) do Hospital das Clínicas da Faculdade de Medicina da Universidade de São Paulo.

5. Médico Cardiologista Diretor da Unidade Clínica de Emergência - InCor - HCFMUSP. Unidade Clínica de Emergência - Instituto do Coração (InCor) do Hospital das Clínicas da Faculdade de Medicina da Universidade de São Paulo.

Endereço para correspondência: Alexandre de Matos Soeiro. Av. Dr. Eneas de Carvalho Aguiar, 44 - Cerqueira César. São Paulo, SP, Brasil. CEP: 05403-900. E-mail: alexandre.soeiro@cardiol.br 


\section{INTRODUÇÃO}

displasia arritmogênica de ventrículo direito
(DAVD) trata-se de uma miocardiopatia
geneticamente determinada caracterizada por substituição fibrogordurosa do miocárdio do ventrículo direito. As manifestações fenotípicas e consequente diagnóstico costumam ser precoces ainda na adolescência ou no adulto jovem ${ }^{1,2}$, sendo uma das principais causas de morte súbita em indivíduos jovens ${ }^{1,2}$. Nesse contexto, o diagnóstico em doente sexagenário sem história familiar sugestiva ou sintomas prévios e com possibilidade de tratamento torna esse caso único.

\section{RELATO DO CASO}

Paciente de 62 anos do sexo masculino compareceu ao serviço de emergência devido quadro de palpitações e episódios de pré-síncope nos últimos dois dias. Referia antecedente de hipertensão arterial sistêmica não tratada e tabagismo. Não utilizava medicações.
Ao exame físico apresentava perfusão periférica lentificada, frequência cardíaca $=180$ batimentos por minuto, pressão arterial de 80×50 mmHg, murmúrios vesiculares presentes bilateralmente sem ruídos adventícios e presença de bulhas taquicárdicas, normofonéticas e sem sopros.

Eletrocardiograma mostrava taquicardia ventricular com frequencia cardíaca de 216 batimentos/minuto, QRS largo com duração aproximada de $140 \mathrm{~ms}$ e eixo em torno de $+60^{\circ}$ no plano frontal, voltando-se para posterior no plano horizontal (negativo em V1), sugerindo típica morfologia de via de saída de ventrículo direito. Ainda é possível observar-se dissociação atrioventricular em V6 (Figura 1). Radiografia de tórax sem alterações. Realizada hipótese diagnóstica taquicardia ventricular instável, sendo o paciente submetido à cardioversão elétrica sincronizada com 100 joules com sucesso. Após reversão para ritmo sinusal, o eletrocardiograma mostrava freqüência cardíaca de 96 batimentos/minuto, QRS com padrão de bloqueio de ramo esquerdo e extrassístoles ventriculares isoladas, porém não apresentava alterações isquêmicas agudas ou presença de onda épsilon (Figura 1).

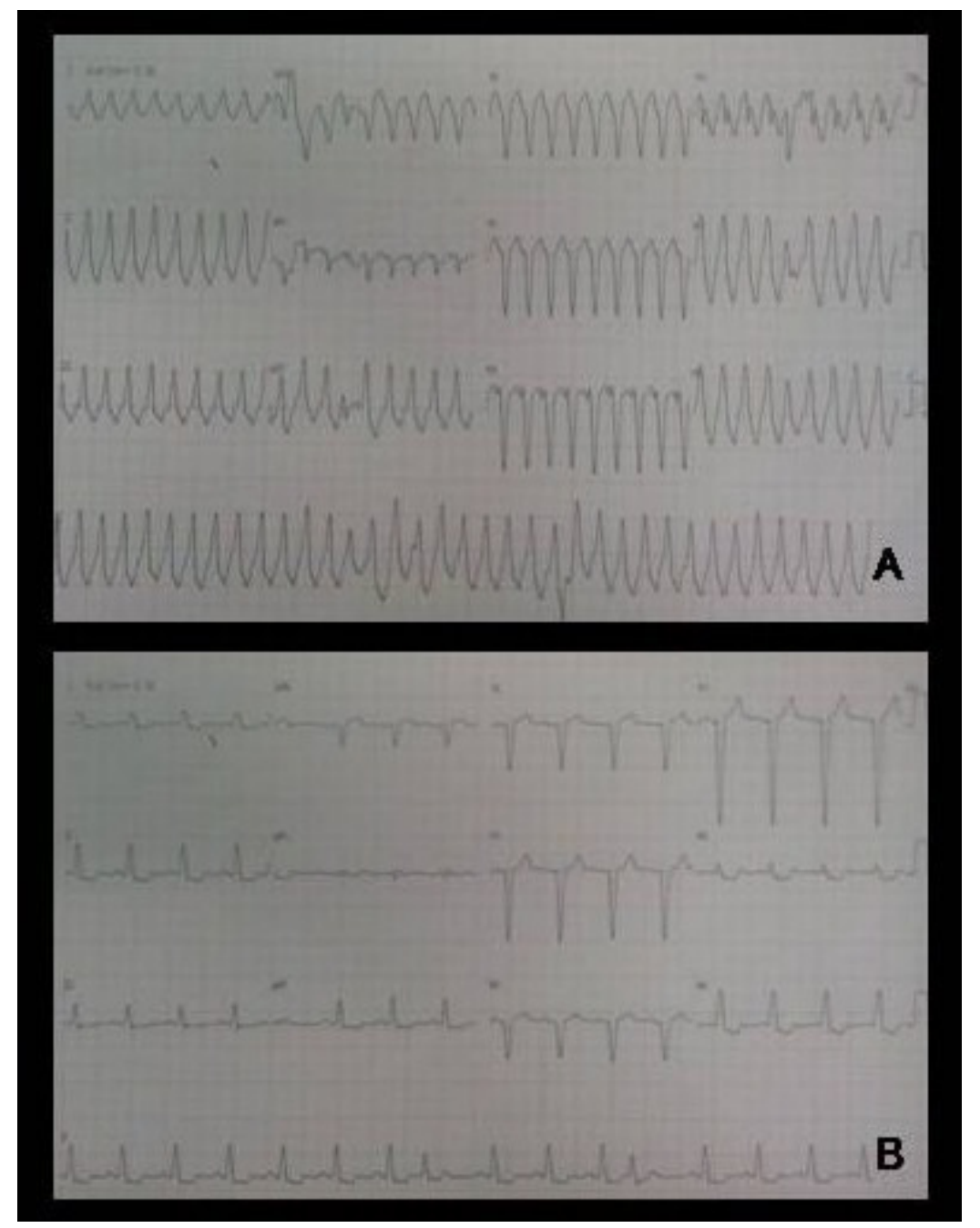

Figura 1 - Eletrocardiogramas durante (A) e após a reversão (B) da taquicardia ventricular 
O paciente apresentou melhora hemodinâmica sendo iniciado o uso de amiodarona $900 \mathrm{mg} /$ dia endovenosa. Exames laboratoriais e marcadores de necrose miocárdica não apresentaram alterações significativas. Ecocardiograma transtorácico mostrou disfunção ventricular discreta com fração de ejeção de ventrículo esquerdo de $52 \%$ e ventrículo esquerdo com diâmetros diastólico e sistólico de 50 e $36 \mathrm{~mm}$, respectivamente. Cineangiocoronariografia não mostrou obstruções coronarianas. Solicitada ressonância magnética cardíaca que mostrou ventrículo direito com dilatação e disfunção moderada/importante com fração de ejeção de $31 \%$ e presença de discinesia e afilamento na via de saída (Figura 2).

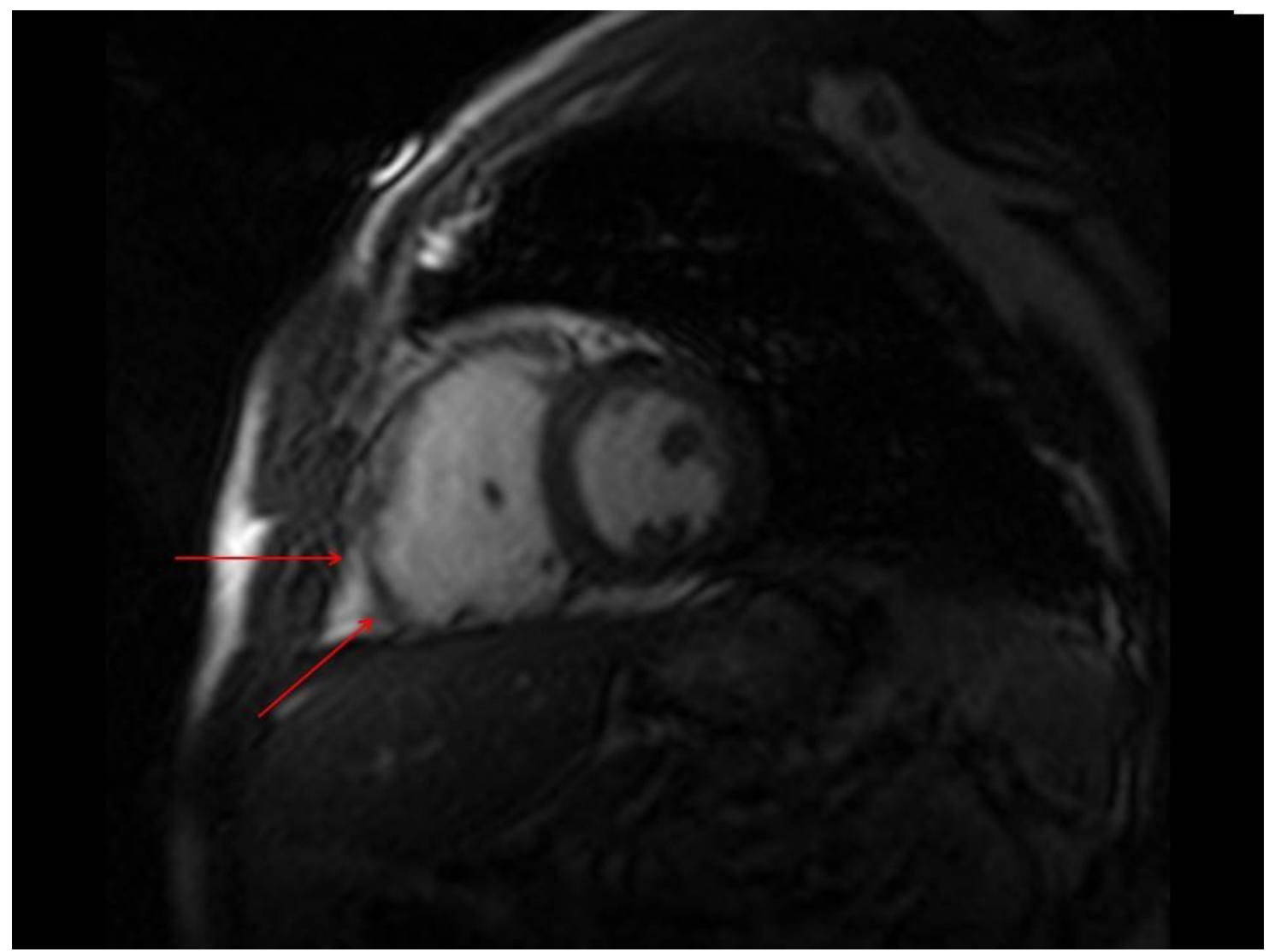

Figura 2 - Ressonância magnética cardíaca mostrando ventrículo direito com dilatação e disfunção moderada/importante e presença de discinesia e afilamento na via de saída (setas vermelhas)

Estes achados sugeriram a hipótese diagnóstica de DAVD, sendo solicitado eletrocardiograma de alta resolução que confirmou a presença de potencial tardio (durações de QRS's abaixo de $40 \mathrm{uV}$ de $42 \mathrm{~ms}$ [normal quando inferior a $35 \mathrm{~ms}$ ] e voltagem dos QRS's nos $40 \mathrm{~ms}$ finais de 14,6 $\mathrm{uV}$ [normal quando maior que $20 \mathrm{uV}$ ]), confirmando a mesma. Devido ao alto risco de morte súbita, foi optado por colocação de cardiodesfibrilador implantável. O paciente evoluiu sem intercorrências e recebeu alta hospitalar após 24 dias em uso de amiodarona $600 \mathrm{mg} / \mathrm{dia}$.

\section{DISCUSSÃO}

De acordo com o último Task Force publicado em 1994 e revisado em 2010 que define os critérios diagnósticos da DAVD, ${ }^{2}$ são necessários dois critérios maiores ou um critério maior e dois menores ou quatro menores para definição do diagnóstico. A presença de achados como alteração morfológica específica na ressonância magnética cardíaca (um critério maior), taquicardia ventricular com morfologia de via de saída de ventrículo direito, bloqueio de ramo esquerdo no eletrocardiograma basal e presença de potencial tardio no eletrocardiograma de alta resolução (três critérios menores), levam à definição da DAVD nesse caso.

A manifestação clínica da doença é variável. Em fase precoce geralmente os indivíduos são assintomáticos, porém já apresentam risco elevado de morte súbita, tanto no esforço como no repouso. A suspeita clínica deve ocorrer nos pacientes com queixas de taquicardia, palpitações, fadiga e que tenham história familiar positiva para morte súbita precoce em parentes de primeiro e segundo graus. À medida que a doença progride, surgem arritmias e sintomas de palpitações e síncopes, fase na qual se encon- 
trava o doente em questão. Em último estágio, o paciente pode evoluir com disfunção biventricular e sintomas de insuficiência cardíaca predominante sobre o componente $\operatorname{arritmogênico~}^{1,2}$.

Conforme observado no caso, o ecocardiograma transtorácico não havia mostrado alterações em ventrículo direito visualizadas posteriormente na ressonância magnética. Isso se deve ao fato, principalmente, de tratar-se de um método examinador dependente, passível de erros de interpretação. Ao contrário, a ressonância é considerada por muitos autores o exame ideal para diagnóstico, permitindo análise criteriosa e detalhadas dos ventrículos e da infiltração gordurosa presente na DAVD ${ }^{1,2}$.

A DAVD é uma das principais causas de morte súbita em indivíduos jovens, sendo esta sua forma inicial de apresentação em até $27 \%$ dos pacientes. Seu diagnóstico geralmente é precoce, ainda em fase de juventude, com idade média de 26 anos. História familiar de DAVD é observada em $30 \%$ dos pacientes. ${ }^{3,4}$ Existem poucos relatos na literatura envolvendo indivíduos com idade avançada, sendo rara sua manifestação acima dos sessenta anos de idade.

Observamos 6 casos na literatura de DAVD em pacientes idosos. Diferente do caso apresentado, em 5 destes, o diagnóstico foi confirmado somente na autópsia, sem possibilidade de tratamento. ${ }^{4-7}$ Em um dos casos descritos, o paciente permaneceu 27 anos com sintomas de insuficiência cardíaca e apresentando taquicardias ventriculares, sendo feito o diagnóstico somente após a morte do mesmo através do exame anatomopatológico. Esse fato torna-se relevante e infere a importância de prosseguir a investigação independente da idade do paciente, podendo mudar o tratamento e prognóstico da doença.

Além disso, na maioria dos casos, havia descrição de história familiar de morte súbita ou DAVD comprovada na família, achado este que pode facilitar o diagnóstico da doença. ${ }^{4-7}$ No entanto, no caso descrito não havia descrição de tal fato, situação esta que dificulta o progresso da investigação diagnóstica. Nesse contexto, a realização de ressonância magnética cardíaca em casos não esclarecidos de disfunção ventricular e/ou taquicardias ventriculares torna-se fundamental em definir o diagnóstico ou descartar outras causas de miocardiopatias como miocardiopatia dilatada idiopática, sarcoidose, miocárdio não compactado e miocardites agudas ${ }^{1,2}$. Nesse caso apresentado, a ressonância foi capaz de descartar os diagnósticos diferenciais acima descritos e corroborar fortemente a hipótese de DAVD. Cabe ressaltar que em todo o indivíduo sexagenário em vigência de taquicardia ventricular, um dos principais diagnósticos a ser descartado é isquemia miocárdica, nesse caso avaliada pela cineangiocoronariografia sem lesões coronárias obstrutivas. Dessa forma, todos os pacientes com disfunção ventricular e/ou arritmias ventriculares não esclarecidas por doenças como isquemia miocárdica, Chagas, doença valvar, etc, idealmente devem ser submetidos à realização de ressonância magnética cardíaca para esclarecimento diagnóstico.

\section{CONCLUSÃO}

O diagnóstico de DAVD em paciente sexagenário mostra que mesmo em indivíduos nessa faixa etária, a realização de ressonância magnética cardíaca em casos de disfunção ventricular e/ou arritmias ventriculares de causa não esclarecida, pode auxiliar no diagnóstico e mudar seu prognóstico e sua forma de tratamento. O diagnóstico deve ser considerado mesmo quando não houver história familiar sugestiva da doença.

\section{REFERÊNCIAS}

1. Ellinor PT, MacRae CA, Thierfelder L. Arrhythmogenic right ventricular cardiomyopathy. Heart Fail Clin. 2010;6(2):16177. doi: 10.1016/j.hfc.2009.12.003.

2. Marcus FI, McKenna WJ, Sherrill D, Basso C, Bauce B, Bluemke DA, et al. Diagnosis of arrhythmogenic right ventricular cardiomyopathy/dysplasia. Eur Heart J. 2010;31(7):806-14. doi: 10.1093/eurheartj/ehq025.

3. Muthappan P, Calkins H. Arrhythmogenic right ventricular dysplasia. Prog Cardiovasc Dis. 2008;51:31-43. doi: 10.1016/j.pcad.2008.01.002.

4. Garcia-Quintana A, Ortega-Trujillo JR, Medina FernandezAceytuno A. Arrhythmogenic right ventricular dysplasia in the elderly. Rev Esp Cardiol. 2007;60(1):87-9. DOI: 10.1016/

\section{S1885-5857(07)60115-7}

5. Kamide K, Satoh S, Okamoto K, Ueda T, Katoh H, Nakano S, Katosh O, Kobayasgi T, Matsuda H, Shibata N. A case of arrhythmogenic right ventricular dysplasia with left and right ventricular hypofunction in an elderlysubject long term followup. Jpn Circ J. 1997;61(10):872-6. http://dx.doi.org/10.1253/ jcj.61.872

6. More D, O'Brien K, Shaw J. Arrhythmogenic right ventricular dysplasia in the elderly. Pacing Clin Electrophysiol. 2002;25(8):1266-9. http://goo.gl/ntjey0

7. Ferreira AC, Garcia SA, Pasquale MA, Canoniero MJ, Peter A. Arrhythmogenic right ventricular dysplasia in the elderly. Heart Dis. 2003;5(6):393-6. 